\title{
The genome sequence of a snail-killing fly, Coremacera
}

\section{marginata (Fabricius, 1775) [version 1; peer review: 2}

\section{approved]}

\author{
Olga Sivell (D1, Duncan Sivell1, Natural History Museum Genome Acquisition Lab, \\ Darwin Tree of Life Barcoding collective, \\ Wellcome Sanger Institute Tree of Life programme, \\ Wellcome Sanger Institute Scientific Operations: DNA Pipelines collective, \\ Tree of Life Core Informatics collective, Darwin Tree of Life Consortium \\ ${ }^{1}$ Department of Life Sciences, Natural History Museum, London, UK
}

\begin{tabular}{l}
\hline V1 First published: 13 Dec 2021, 6:338 \\
https://doi.org/10.12688/wellcomeopenres.17482.1 \\
Latest published: 13 Dec 2021, 6:338 \\
https://doi.org/10.12688/wellcomeopenres.17482.1 \\
\hline
\end{tabular}

\section{Abstract}

We present a genome assembly from an individual female Coremacera marginata (Arthropoda; Insecta; Diptera; Sciomyzidae). The genome sequence is 980 megabases in span. The majority of the assembly $(99.84 \%)$ is scaffolded into six chromosomal pseudomolecules, with the $X$ sex chromosome assembled.

\section{Keywords}

Coremacera marginata, genome sequence, chromosomal, Diptera

This article is included in the Tree of Life gateway.

Open Peer Review
Approval Status
version 1
13 Dec 2021
1. Liping Yan
Beijing, China
2. Craig Wilding (iD), Liverpool John Moores
University, Liverpool, UK
Any reports and responses or comments on the
article can be found at the end of the article.


Corresponding author: Darwin Tree of Life Consortium (mark.blaxter@sanger.ac.uk)

Author roles: Sivell O: Investigation, Resources, Writing - Original Draft Preparation, Writing - Review \& Editing; Sivell D: Investigation, Resources;

Competing interests: No competing interests were disclosed.

Grant information: This work was supported by Wellcome through core funding to the Wellcome Sanger Institute (206194) and the Darwin Tree of Life Discretionary Award (218328).

The funders had no role in study design, data collection and analysis, decision to publish, or preparation of the manuscript.

Copyright: (c) 2021 Sivell O et al. This is an open access article distributed under the terms of the Creative Commons Attribution License, which permits unrestricted use, distribution, and reproduction in any medium, provided the original work is properly cited.

How to cite this article: Sivell O, Sivell D, Natural History Museum Genome Acquisition Lab et al. The genome sequence of a snailkilling fly, Coremacera marginata (Fabricius, 1775) [version 1; peer review: 2 approved] Wellcome Open Research 2021, 6:338 https://doi.org/10.12688/wellcomeopenres.17482.1

First published: 13 Dec 2021, 6:338 https://doi.org/10.12688/wellcomeopenres.17482.1 


\section{Species taxonomy}

Eukaryota; Metazoa; Ecdysozoa; Arthropoda; Hexapoda; Insecta; Pterygota; Neoptera; Endopterygota; Diptera; Brachycera; Muscomorpha; Sciomyzoidea; Sciomyzidae; Coremacera; Coremacera marginata (Fabricius, 1775) (NCBI:txid1226616).

\section{Background}

Sciomyzidae (Diptera) are commonly known as snail-killing flies or marsh flies, the latter name reflecting the habitat preference of many species from this family. Coremacera marginata (Diptera, Sciomyzidae) is a dark grey-brown fly with a characteristic wing pattern consisting of a strongly infuscated wing margin (darker near the coastal vein) and dark brown base colour with numerous pale spots across the rest of the wing. The species is fairly common and widely distributed in England and Wales, in Scotland it has only been recorded from around the Moray and Dornoch Firths (Ball, 2017). It prefers open and dry habitats, particularly calcareous grasslands, also coastal dunes, open scrubby woods, old fields on woodland margins and is occasionally found in wetland habitats (Ball, 2017; Rozkošný, 1984). Flight period occurs from mid-May till beginning of October (Ball, 2017; Speight \& Knutson, 2012).

Coremacera marginata is oviparous. The eggs are laid on or near the host. The larvae are parasitoids of terrestrial snails (Knutson, 1970; Rozkošný, 1984; Rozkošný, 1987), with a preference of Cochlicopa and Discus species in laboratory conditions (Knutson, 1973; Rozkošný, 1984). Upon hatching the larva feeds on a living snail. The host survives for up to ten days, unless infested with multiple larvae (up to 11 have been reported to attack a single snail), in which case death can occur within 24 hours (Knutson, 1973; Rozkošný, 1984). The larva continues to feed on the decomposing tissues until it reaches the second or third instar. It then moves to a second snail to continue feeding, killing the host in one to two days. Rarely, the larva will require a third snail to complete its development. Pupation occurs outside the shell. The larval stage lasts from 22 to 97 days with an average of 52 days, and the pupa from 47 to 124 days (Knutson, 1973; Rozkošný, 1984). This species overwinters as a mature larva or as a pupa (Ball, 2017; Speight \& Knutson, 2012). Adults feed on flowers, dead insects and snails, and also on insect eggs and live snails' secretions (Berg \& Knutson, 1978). First and third larval instars and the puparium have been described by Knutson (1973).

Coremacera marginata was split into two subspecies, Coremacera marginata marginata (Fabricius, 1775) and Coremacera marginata pontica, by Elberg (1968) based on paler specimens from southern European Russia and Iran. This was subsequently rejected by Knutson (1973) due to a lack of differentiating structural characters that would support the separation.

The high-quality genome sequence described here is the first one reported for Coremacera marginata and has been generated as part of the Darwin Tree of Life project. It will aid in understanding the biology, physiology and ecology of the species.

\section{Genome sequence report}

The genome was sequenced from a single female $C$. marginata (Figure 1) collected from Wigmore Park, Luton, UK (latitude 51.88378, longitude -0.36861422 ). A total of 25-fold coverage in Pacific Biosciences single-molecule long reads and 33fold coverage in $10 \mathrm{X}$ Genomics read clouds were generated. Primary assembly contigs were scaffolded with chromosome conformation Hi-C data. Manual assembly curation corrected 617 missing/misjoins and removed 8 haplotypic duplications, reducing the assembly size by $0.18 \%$ and the scaffold number by $82.91 \%$, and increasing the scaffold N50 by $268.04 \%$.

The final assembly has a total length of $980 \mathrm{Mb}$ in 60 sequence scaffolds with a scaffold N50 of $184.1 \mathrm{Mb}$ (Table 1). The majority, $99.84 \%$, of the assembly sequence was assigned to 6 chromosomal-level scaffolds, representing 5 autosomes (numbered by sequence length), and the $\mathrm{X}$ sex chromosome (Figure 2-Figure 5; Table 2). The assembly has a BUSCO v5.1.2 (Manni et al., 2021) completeness of 97.2\% (single $96.2 \%$, duplicated $1.1 \%$ ) using the diptera_odb10 reference set. While not fully phased, the assembly deposited is of one haplotype. Contigs corresponding to the second haplotype have also been deposited.

\section{Methods}

Sample acquisition and nucleic acid extraction

A female C. marginata (idCorMarg1) was collected from Wigmore Park, Luton, UK (latitude 51.88378, longitude 0.36861422 ) by Olga Sivell, Natural History Museum, and identified by Duncan Sivell, Natural History Museum based on Rozkošný (1984) and Ball (2017). The specimens were collected using a net and snap-frozen on dry ice.

DNA was extracted at the Tree of Life laboratory, Wellcome Sanger Institute. The idCorMarg1 sample was weighed and dissected on dry ice with tissue set aside for $\mathrm{Hi}-\mathrm{C}$ and RNA sequencing. Thorax tissue was cryogenically disrupted

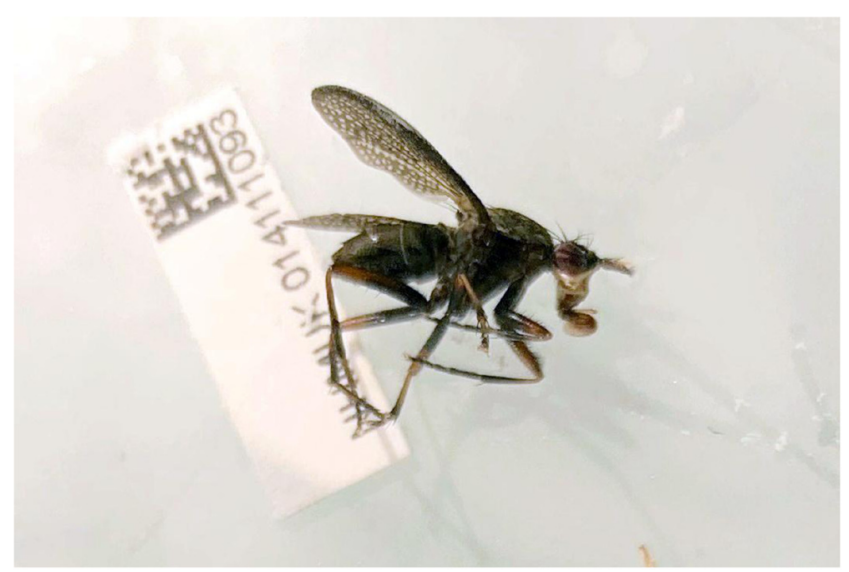

Figure 1. Image of the idCorMarg1 specimen taken during preservation and processing. 


\begin{tabular}{|c|c|}
\hline \multicolumn{2}{|l|}{ Project accession data } \\
\hline Assembly identifier & idCorMarg1.1 \\
\hline Species & Coremacera marginata \\
\hline Specimen & idCorMarg1 \\
\hline NCBI taxonomy ID & 1226616 \\
\hline BioProject & PRJEB45188 \\
\hline BioSample ID & SAMEA7521524 \\
\hline Isolate information & $\begin{array}{l}\text { Female, thorax (genome assembly), head } \\
\text { (Hi-C), abdomen (RNA-Seq) }\end{array}$ \\
\hline \multicolumn{2}{|l|}{ Raw data accessions } \\
\hline PacificBiosciences SEQUEL II & ERR6412041 \\
\hline 10X Genomics Illumina & ERR6054930-ERR6054933 \\
\hline Hi-C Illumina & ERR6054934 \\
\hline Illumina polyA RNA-Seq & ERR6688408 \\
\hline \multicolumn{2}{|l|}{ Genome assembly } \\
\hline Assembly accession & GCA_914767935.1 \\
\hline Accession of alternate haplotype & GCA_914767655.1 \\
\hline Span (Mb) & 980 \\
\hline Number of contigs & 889 \\
\hline Contig N50 length (Mb) & 2.7 \\
\hline Number of scaffolds & 60 \\
\hline Scaffold N50 length (Mb) & 184.1 \\
\hline Longest scaffold (Mb) & 252.6 \\
\hline BUSCO* genome score & $\begin{array}{l}\text { C:97.2\%[S:96.2\%,D:1.1\%],F:0.7\%,M:2.1\%, } \\
\text { n:3285 }\end{array}$ \\
\hline
\end{tabular}

*BUSCO scores based on the diptera_odb10 BUSCO set using v5.1.2. C= complete [S= single copy, $D=$ duplicated], F=fragmented, $M=$ missing, $n=$ number of orthologues in comparison. A full set of BUSCO scores is available at https://blobtoolkit.genomehubs. org/view/idCorMarg1.1/dataset/CAJZBS01/busco.

to a fine powder using a Covaris cryoPREP Automated Dry Pulveriser, receiving multiple impacts. Fragment size analysis of 0.01-0.5 ng of DNA was then performed using an Agilent FemtoPulse. High molecular weight (HMW) DNA was extracted using the Qiagen MagAttract HMW DNA extraction kit. Low molecular weight DNA was removed from a 200-ng aliquot of extracted DNA using 0.8X AMpure XP purification kit prior to $10 \mathrm{X}$ Chromium sequencing; a minimum of $50 \mathrm{ng}$ DNA was submitted for $10 \mathrm{X}$ sequencing. HMW DNA was sheared into an average fragment size between $12-20 \mathrm{~kb}$ in a Megaruptor 3 system with speed setting 30. Sheared DNA was purified by solid-phase reversible immobilisation using AMPure $\mathrm{PB}$ beads with a $1.8 \mathrm{X}$ ratio of beads to sample to remove the shorter fragments and concentrate the DNA sample. The concentration of the sheared and purified DNA was assessed using a Nanodrop spectrophotometer and Qubit Fluorometer and Qubit dsDNA High Sensitivity Assay kit. Fragment size distribution was evaluated by running the sample on the FemtoPulse system.

RNA was extracted from abdomen tissue in the Tree of Life Laboratory at the WSI using TRIzol (Invitrogen), according to the manufacturer's instructions. RNA was then eluted in $50 \mu \mathrm{l}$ RNAse-free water and its concentration RNA assessed using a Nanodrop spectrophotometer and Qubit Fluorometer using the Qubit RNA Broad-Range (BR) Assay kit. Analysis 


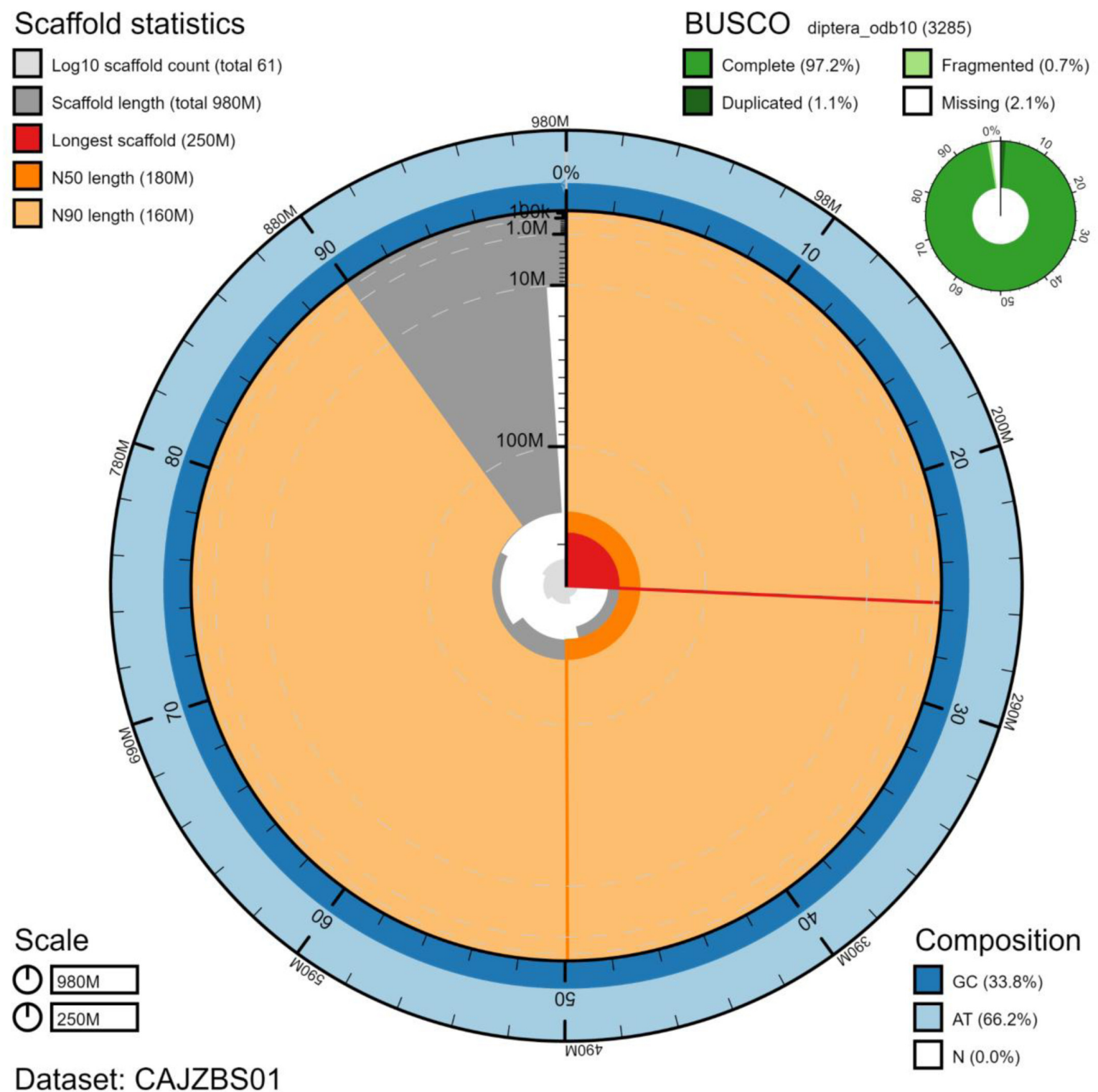

Figure 2. Genome assembly of Coremacera marginata, idCorMarg1.1: metrics. The BlobToolKit Snailplot shows N50 metrics and BUSCO gene completeness. The main plot is divided into 1,000 size-ordered bins around the circumference with each bin representing $0.1 \%$ of the $979,680,483 \mathrm{bp}$ assembly. The distribution of chromosome lengths is shown in dark grey with the plot radius scaled to the longest chromosome present in the assembly $(252,586,203 \mathrm{bp}$, shown in red). Orange and pale-orange arcs show the N50 and N90 chromosome lengths $(184,110,224$ and $162,576,309$ bp), respectively. The pale grey spiral shows the cumulative chromosome count on a log scale with white scale lines showing successive orders of magnitude. The blue and pale-blue area around the outside of the plot shows the distribution of GC, AT and N percentages in the same bins as the inner plot. A summary of complete, fragmented, duplicated and missing BUSCO genes in the diptera_odb10 set is shown in the top right. An interactive version of this figure is available at https://blobtoolkit.genomehubs.org/ view/idCorMarg1.1/dataset/CAJZBS01/snail.

of the integrity of the RNA was done using Agilent RNA 6000 Pico Kit and Eukaryotic Total RNA assay.

\section{Sequencing}

Pacific Biosciences HiFi circular consensus and 10X Genomics read cloud DNA sequencing libraries were constructed according to the manufacturers' instructions. Poly(A) RNA-Seq libraries were constructed using the NEB Ultra II RNA Library Prep kit. DNA and RNA sequencing was performed by the Scientific Operations core at the WSI on Pacific Biosciences SEQUEL II (HiFi), Illumina HiSeq $X(10 X)$ and Illumina HiSeq 4000 (RNA-Seq) instruments. Hi-C data were generated 


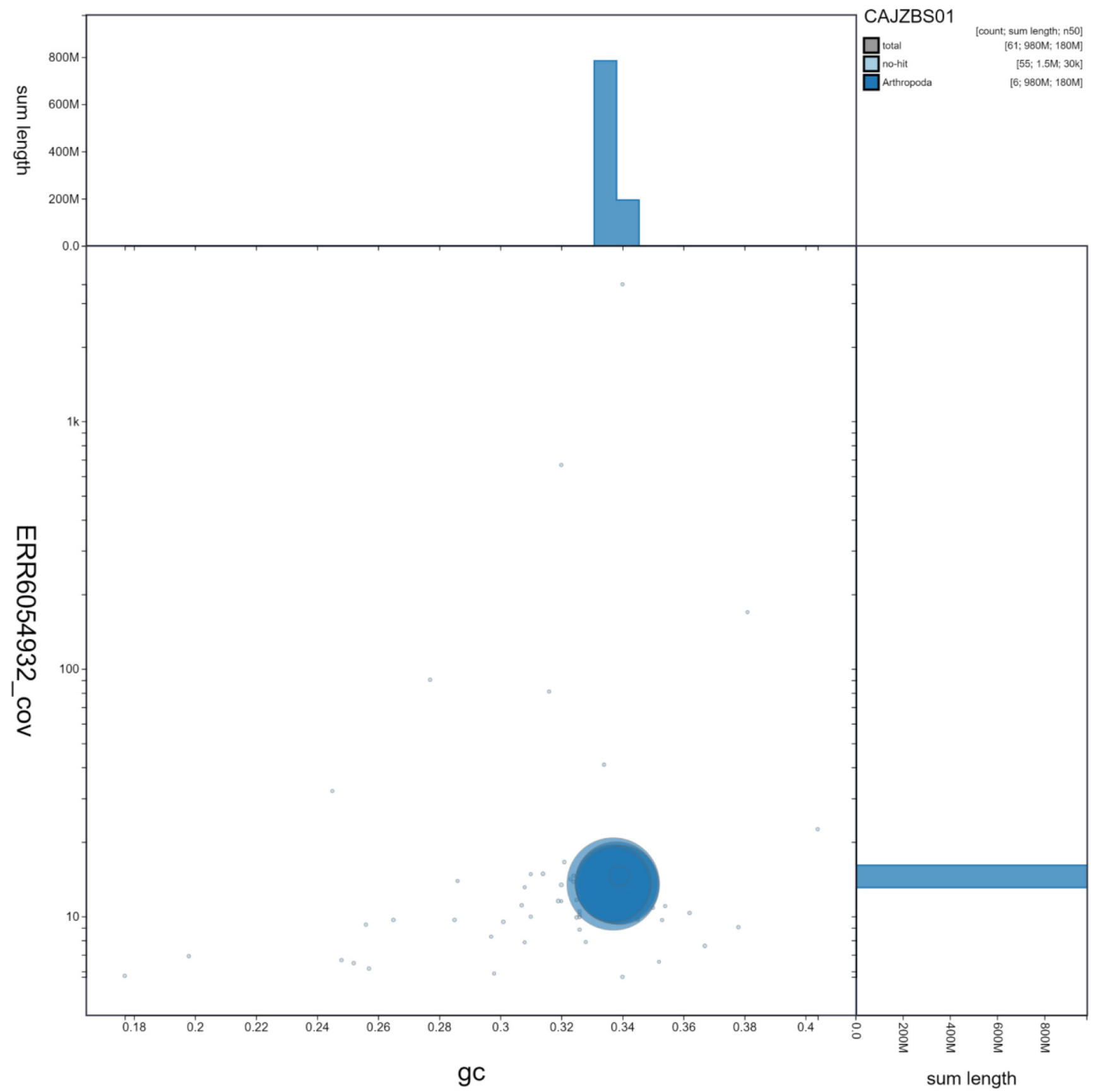

Figure 3. Genome assembly of Coremacera marginata, idCorMarg1.1: GC coverage. BlobToolKit GC-coverage plot. Scaffolds are coloured by phylum. Circles are sized in proportion to scaffold length. Histograms show the distribution of scaffold length sum along each axis. An interactive version of this figure is available at https://blobtoolkit.genomehubs.org/view/idCorMarg1.1/dataset/CAJZBS01/blob.

from abdomen tissue of the same specimen using the Arima $\mathrm{Hi}-\mathrm{C}+\mathrm{kit}$ and sequenced on an Illumina NovaSeq 6000 instrument.

\section{Genome assembly}

Assembly was carried out with Hifiasm (Cheng et al., 2021); haplotypic duplication was identified and removed with 


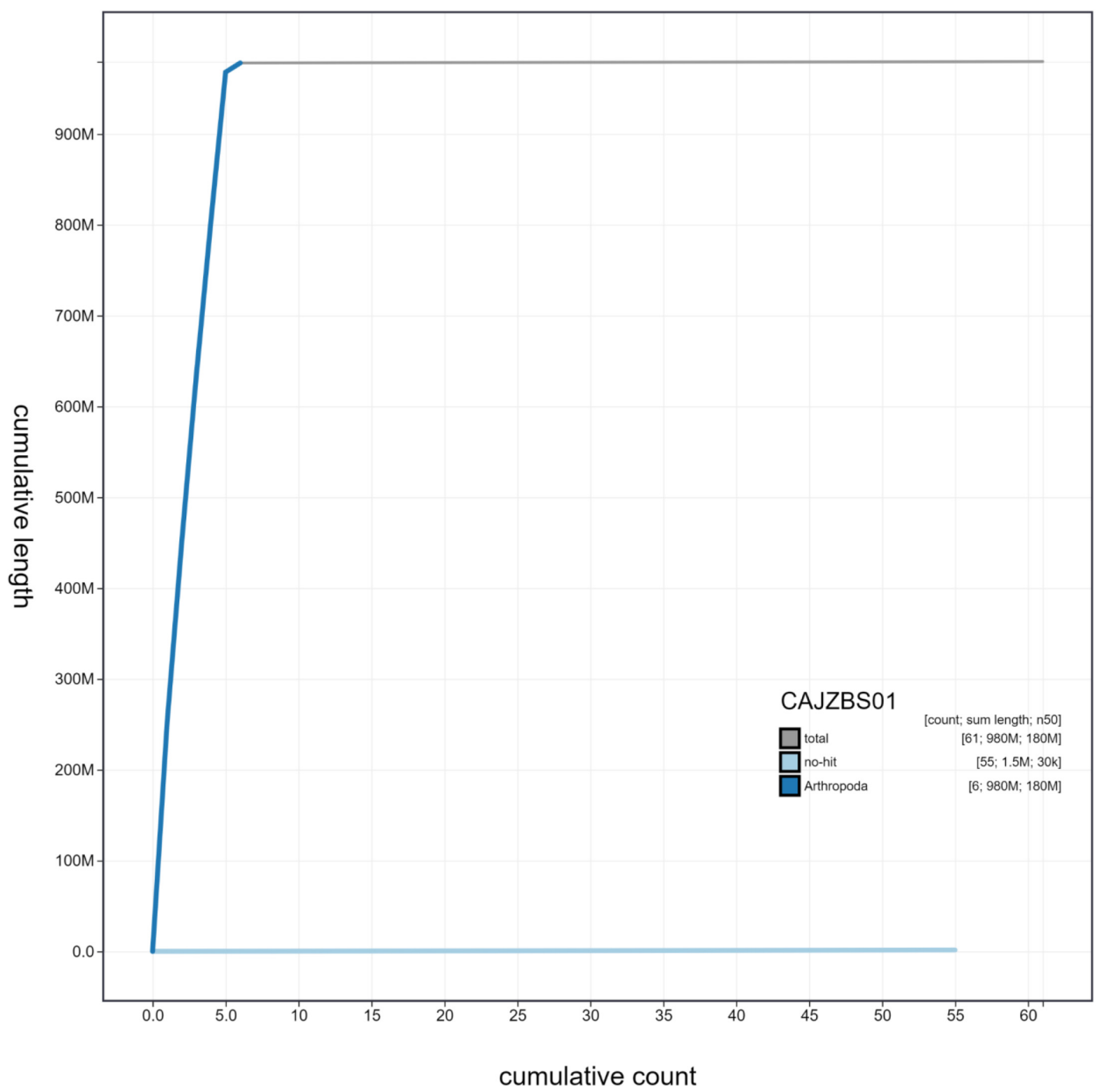

Figure 4. Genome assembly of Coremacera marginata, idCorMarg1.1: cumulative sequence. BlobToolKit cumulative sequence plot. The grey line shows cumulative length for all scaffolds. Coloured lines show cumulative lengths of scaffolds assigned to each phylum using the buscogenes taxrule. An interactive version of this figure is available at https://blobtoolkit.genomehubs.org/view/idCorMarg1.1/dataset/ CAJZBS01/cumulative.

purge_dups (Guan et al., 2020). One round of polishing was performed by aligning $10 \mathrm{X}$ Genomics read data to the assembly with longranger align, calling variants with freebayes (Garrison \& Marth, 2012). The assembly was then scaffolded with Hi-C data (Rao et al., 2014) using SALSA2
(Ghurye et al., 2019). The assembly was checked for contamination as described previously (Howe et al., 2021). Manual curation (Howe et al., 2021) was performed using HiGlass (Kerpedjiev et al., 2018) and Pretext. The mitochondrial genome was assembled using MitoHiFi (Uliano-Silva et al., 2021), 


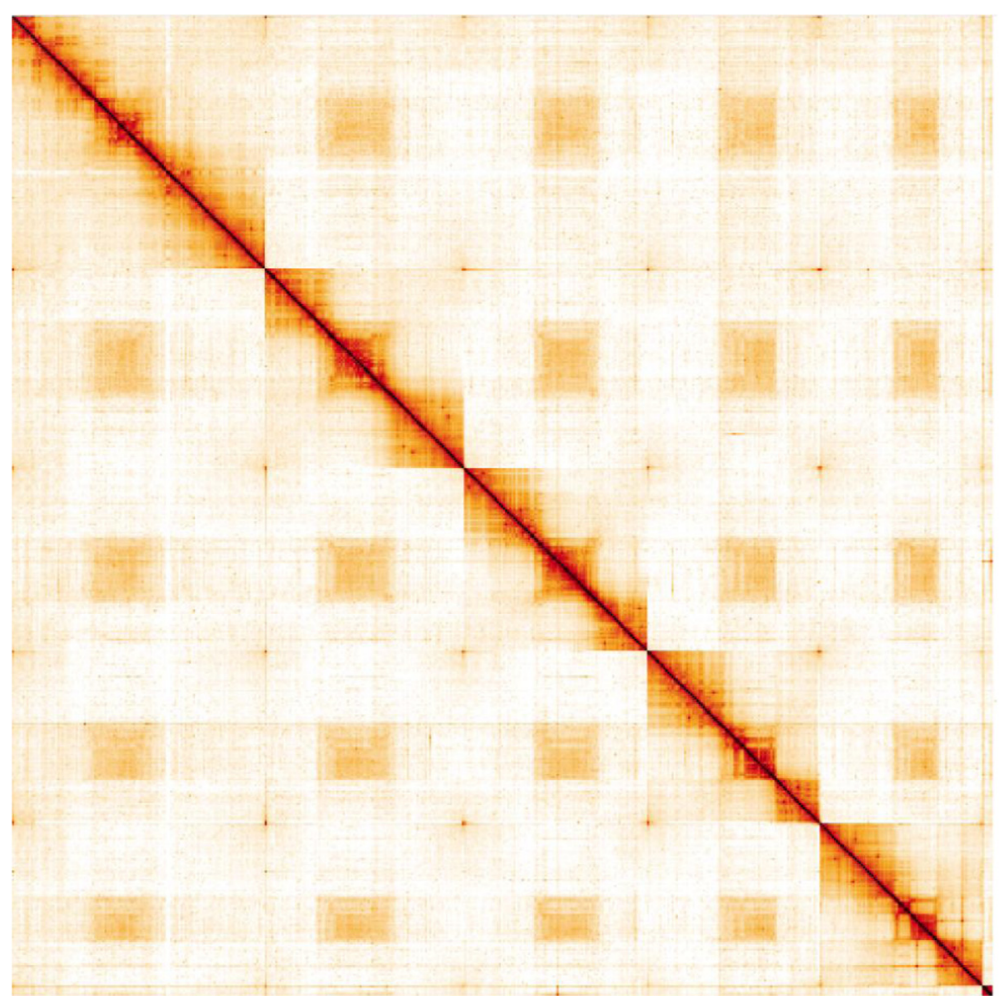

Figure 5. Genome assembly of Coremacera marginata, idCorMarg1.1: Hi-C contact map. Hi-C contact map of the idCorMarg1.1 assembly, visualised in HiGlass. Chromosomes are given in order of size, from left to right and top to bottom.

Table 2. Chromosomal pseudomolecules in the genome assembly of Coremacera marginata, idCorMarg1.1.

\begin{tabular}{|c|c|c|c|}
\hline INSDC accession & Chromosome & Size (Mb) & GC\% \\
\hline OU612043.1 & 1 & 252.59 & 33.7 \\
\hline OU612044.1 & 2 & 198.14 & 33.8 \\
\hline OU612045.1 & 3 & 184.11 & 33.9 \\
\hline OU612046.1 & 4 & 170.64 & 33.7 \\
\hline OU612047.1 & 5 & 162.58 & 33.7 \\
\hline OU612048.1 & $X$ & 10.12 & 33.9 \\
\hline OU612049.1 & MT & 0.02 & 34.6 \\
\hline- & Unplaced & 1.49 & 31.4 \\
\hline
\end{tabular}

which performed annotation using MitoFinder (Allio et al., 2020). The genome was analysed and BUSCO scores generated within the BlobToolKit environment (Challis et al., 2020). Table 3 contains a list of all software tool versions used, where appropriate.
Table 3. Software tools used.

\begin{tabular}{|c|c|c|}
\hline $\begin{array}{l}\text { Software } \\
\text { tool }\end{array}$ & Version & Source \\
\hline Hifiasm & 0.12 & Cheng et al., 2021 \\
\hline $\begin{array}{l}\text { purge } \\
\text { dups }\end{array}$ & 1.2 .3 & Guan et al., 2020 \\
\hline SALSA2 & 2.2 & Ghurye et al., 2019 \\
\hline $\begin{array}{l}\text { longranger } \\
\text { align }\end{array}$ & 2.2 .2 & $\begin{array}{l}\text { https:// } \\
\text { support.10xgenomics.com/ } \\
\text { genome-exome/software/ } \\
\text { pipelines/latest/advanced/ } \\
\text { other-pipelines }\end{array}$ \\
\hline freebayes & 1.3.1-17-gaa2ace8 & Garrison \& Marth, 2012 \\
\hline MitoHiFi & 2.0 & Uliano-Silva et al., 2021 \\
\hline gEVAL & N/A & Chow et al., 2016 \\
\hline HiGlass & 1.11 .6 & Kerpedjiev et al., 2018 \\
\hline PretextView & $0.2 . x$ & $\begin{array}{l}\text { https://github.com/wtsi- } \\
\text { hpag/PretextView }\end{array}$ \\
\hline BlobToolKit & 2.6 .2 & Challis et al., 2020 \\
\hline
\end{tabular}




\section{Ethics/compliance issues}

The materials that have contributed to this genome note have been supplied by a Darwin Tree of Life Partner. The submission of materials by a Darwin Tree of Life Partner is subject to the Darwin Tree of Life Project Sampling Code of Practice. By agreeing with and signing up to the Sampling Code of Practice, the Darwin Tree of Life Partner agrees they will meet the legal and ethical requirements and standards set out within this document in respect of all samples acquired for, and supplied to, the Darwin Tree of Life Project. Each transfer of samples is further undertaken according to a Research Collaboration Agreement or Material Transfer Agreement entered into by the Darwin Tree of Life Partner, Genome Research Limited (operating as the Wellcome Sanger Institute), and in some circumstances other Darwin Tree of Life collaborators.

\section{Data availability}

European Nucleotide Archive: Coremacera marginata. Accession number PRJEB45188: https://www.ebi.ac.uk/ena/browser/view/ PRJEB45188.

The genome sequence is released openly for reuse. The C. marginata genome sequencing initiative is part of the Darwin Tree of Life (DToL) project. All raw sequence data and the assembly have been deposited in INSDC databases.
The genome will be annotated using the RNA-Seq data and presented through the Ensembl pipeline at the European Bioinformatics Institute. Raw data and assembly accession identifiers are reported in Table 1.

\section{Author information}

Members of the Natural History Museum Genome Acquisition Lab are listed here: https://doi.org/10.5281/zenodo.5746819.

Members of the Darwin Tree of Life Barcoding collective are listed here: https://doi.org/10.5281/zenodo.5744972.

Members of the Wellcome Sanger Institute Tree of Life programme are listed here: https://doi.org/10.5281/zenodo. 5744840 .

Members of Wellcome Sanger Institute Scientific Operations: DNA Pipelines collective are listed here: https://doi.org/10.5281/ zenodo. 5746904

Members of the Tree of Life Core Informatics collective are listed here: https://doi.org/10.5281/zenodo.5743293.

Members of the Darwin Tree of Life Consortium are listed here: https://doi.org/10.5281/zenodo.5638618.
Allio R, Schomaker-Bastos A, Romiguier J, et al.: MitoFinder: Efficient Automated Large-Scale Extraction of Mitogenomic Data in Target Enrichment Phylogenomics. Mol Ecol Resour. 2020; 20(4): 892-905. PubMed Abstract | Publisher Full Text | Free Full Text Ball S: The British Sciomyzidae. Dipterists Forum, unpublished identification guide, 2017.

Berg CO, Knutson L: Biology and systematics of the Sciomyzidae. Annu Rev Entomol. 1978; 23(1): 239-258.

Publisher Full Text

Challis R, Richards E, Rajan J, et al:: BlobToolKit - Interactive Quality Assessment of Genome Assemblies. G3 (Bethesda). 2020; 10(4): 1361-74. PubMed Abstract | Publisher Full Text | Free Full Text

Cheng $\mathrm{H}$, Concepcion GT, Feng $\mathrm{X}$, et al:: Haplotype-Resolved de Novo Assembly Using Phased Assembly Graphs with Hifiasm. Nat Methods. 2021 18(2): 170-75.

PubMed Abstract | Publisher Full Text | Free Full Text

Elberg K: Zur Kenntnis der Statinia-Arten aus der UdSSR (Diptera:

Sciomyzidae). Beiträge zur Entomologie. 1968; 18: 663-670.

Publisher Full Text

Garrison E, Marth G: Haplotype-Based Variant Detection from Short-Read Sequencing. arXiv: 1207.3907. 2012.

Reference Source

Ghurye J, Rhie A, Walenz BP, et al.: Integrating Hi-C Links with Assembly Graphs for Chromosome-Scale Assembly. PLoS Comput Biol. 2019; 15(8): e1007273.

PubMed Abstract | Publisher Full Text | Free Full Text

Guan D, McCarthy SA, Wood J, et al.: Identifying and Removing Haplotypic Duplication in Primary Genome Assemblies. Bioinformatics. 2020; 36(9): 2896-98.

PubMed Abstract | Publisher Full Text | Free Full Text

Howe K, Chow W, Collins J, et al.: Significantly Improving the Quality of Genome Assemblies through Curation. GigaScience. 2021; 10(1): giaa153. PubMed Abstract | Publisher Full Text | Free Full Text
Kerpedjiev P, Abdennur N, Lekschas F, et al.: HiGlass: Web-Based Visual Exploration and Analysis of Genome Interaction Maps. Genome Biol. 2018; 19(1): 125.

PubMed Abstract | Publisher Full Text | Free Full Text

Knutson LV: Biology of Snail-Killing Flies in Sweden (Dipt., Sciomyzidae)1. Insect Syst Evol. 1970; 1(4): 307-314.

Reference Source

Knutson LV: Biology and immature stages of Coremacera marginata F. a predator of terrestrial snails (Dipt. Sciomyzidae). Insect Syst Evol. 1973; 4(2): 123-133.

Reference Source

Manni M, Berkeley MR, Seppey M, et al.: BUSCo Update: Novel and Streamlined Workflows along with Broader and Deeper Phylogenetic Coverage for Scoring of Eukaryotic, Prokaryotic, and Viral Genomes. $\mathrm{Mol}$ Biol Evol. 2021; 38(10): 4647-54.

PubMed Abstract | Publisher Full Text | Free Full Text

Rao SSP, Huntley MH, Durand NC, et al:: A 3D Map of the Human Genome at Kilobase Resolution Reveals Principles of Chromatin Looping. Cell. 2014; 159(7): 1665-80.

PubMed Abstract | Publisher Full Text | Free Full Text

Rozkošný R: The Sciomyzidae (Diptera) of Fennoscandia and Denmark. Fauna Entomologica Scandinavica. Brill, 1984; 14.

Reference Source

Rozkošný R: A review of the Palaearctic Sciomyzidae (Diptera). Folia Facultatis Scientiarum Naturalium Universitatis Purkynianae Brunensis Biologia. 1987; 86: 1-156.

Reference Source

Speight MCD, Knutson LV: Species accounts for Sciomyzidae and Phaeomyiidae (Diptera) known from the Atlantic zone of Europe. Dipterists Digest. 2012; 19(1): 1-38.

Uliano-Silva M, Nunes JGF, Krasheninnikova K, et al:: marcelauliano/MitoHiFi: mitohifi_v2.0. 2021

Publisher Full Text 


\section{Open Peer Review}

\section{Current Peer Review Status:}

\section{Version 1}

Reviewer Report 09 November 2022

https://doi.org/10.21956/wellcomeopenres.19332.r53078

(C) 2022 Wilding C. This is an open access peer review report distributed under the terms of the Creative Commons Attribution License, which permits unrestricted use, distribution, and reproduction in any medium, provided the original work is properly cited.

\section{Craig Wilding}

School of Biological and Environmental Sciences, Liverpool John Moores University, Liverpool, UK

This Genome Note provides a useful review of the genome assembly and annotation efforts for Coremacera marginata. Sivell and Sivell have produced an exceptionally high-quality genome for a single female fly. The methodological details are clear and the results well presented. Personally, I think It would be useful to know (in the introduction) what pre-existing genomic or transcriptomic resources exist for the Sciomyzids? Is this the first genome available? Indeed is the genome size typical or exceptional for this group? I assume that as for most other flies the male is heterogametic so it is unfortunate that a male was not sequenced. Perhaps a comment to this effect could be added to the discussion.

RNASeq data are mentioned but there is no indication of the number of gene models identified. Is RNASeq data the only source of gene models or was automatic annotation also used? Perhaps the number of gene models could be added to Table 1 ?

I would also find it interesting to have further details of the manual curation that led to the improvement in scaffold number and scaffold N50.

\section{Is the rationale for creating the dataset(s) clearly described?}

Yes

Are the protocols appropriate and is the work technically sound?

Yes

Are sufficient details of methods and materials provided to allow replication by others? Yes

Are the datasets clearly presented in a useable and accessible format? Yes

Competing Interests: No competing interests were disclosed. 
Reviewer Expertise: Evolutionary genetics and genomics (invertebrates)

I confirm that I have read this submission and believe that I have an appropriate level of expertise to confirm that it is of an acceptable scientific standard.

Reviewer Report 07 April 2022

https://doi.org/10.21956/wellcomeopenres.19332.r49256

(C) 2022 Yan L. This is an open access peer review report distributed under the terms of the Creative Commons Attribution License, which permits unrestricted use, distribution, and reproduction in any medium, provided the original work is properly cited.

\section{Liping Yan}

School of Ecology and Nature Conservation, Beijing Forestry University, Beijing, China

Sivell et al. (2021) sequenced genomic data of the snail-killing fly, Coremacera marginata. The study was well planned, the methodology is fine, and manuscript is well structured and written in general. Although this is a small paper documenting data, I am looking forward to the follow-up studies with further information and the evolutionary story of this interesting fly and its relatives.

Is the rationale for creating the dataset(s) clearly described?

Yes

Are the protocols appropriate and is the work technically sound?

Yes

Are sufficient details of methods and materials provided to allow replication by others? Yes

Are the datasets clearly presented in a useable and accessible format? Yes

Competing Interests: No competing interests were disclosed.

Reviewer Expertise: Phylogenomics, transcriptomics.

I confirm that I have read this submission and believe that I have an appropriate level of expertise to confirm that it is of an acceptable scientific standard. 